\title{
Coexistence of lung alveolar adenoma with cerebral arteriovenous malformations: A case report and literature review
}

\author{
XIAOPING TANG ${ }^{1}$, ZIHAN WU ${ }^{2}$ and YIHONG SHEN ${ }^{1}$ \\ Departments of ${ }^{1}$ Respiratory Medicine and ${ }^{2}$ Pathology, First Affiliated Hospital, \\ College of Medicine, Zhejiang University, Hangzhou, Zhejiang 310003, P.R. China
}

Received August 14, 2014; Accepted April 30, 2015

DOI: $10.3892 / \mathrm{ol} .2015 .3225$

\begin{abstract}
Alveolar adenoma is an extremely rare benign tumor of the lung derived from type II pneumocytes. To date, only a limited number of alveolar adenoma cases have been reported in the literature. These tumors are usually discovered incidentally on radiographic images. However, other tumor types must be excluded when the characteristics of alveolar adenoma are not typical on the radiographic images. Therefore, diagnosis of alveolar adenomas is based on the combination of gross structural analysis and immunohistochemistry. The present study described a case of alveolar adenoma in a 47-year-old female patient. A chest X-ray revealed a pulmonary lesion in the right lower lobe and, subsequently, the patient underwent a right lower lobectomy. Pathological examination of the surgically resected tissue confirmed the initial diagnosis of alveolar adenoma. In addition, intracranial vascular malformations were detected on a computed tomography scan, but the patient did not experience any notable symptoms. Considering the benign nature of the malformations, as determined by digital subtraction angiography, the patient selected to undergo follow-up with observation only. The postoperative course was uneventful and no recurrence of pulmonary lesions was noted after $>4$ years, while the condition of the intracranial lesion was stable. In conclusion, the current study described a unique case of adenoma alveolar coexisting with intracranial vascular malformations. A literature review of previously-reported alveolar adenoma cases was also conducted.
\end{abstract}

\section{Introduction}

Alveolar adenoma is an extremely rare pulmonary neoplasm with specific characteristics, identified upon gross and

Correspondence to: Professor Yihong Shen, Department of Respiratory Medicine, First Affiliated Hospital, College of Medicine, Zhejiang University, 79 Qingchun Road, Hangzhou, Zhejiang 310003, P.R. China

E-mail:drsyh@163.com

Key words: alveolar adenoma, arteriovenous malformation, intracranial lesion microscopic analysis (1,2). Grossly, alveolar adenoma presents a rounded configuration that is well-circumscribed with a spongy gray-white multilocular cut surface (1). Microscopically, this tumor comprises a number of cystic spaces of variable sizes, which are lined by type 2 alveolar epithelial cells (1-3). These tumors are often discovered incidentally on chest X-rays and predominantly occur in the lung periphery (4). The radiological images typically reveal a solitary, well-delineated, non-calcified nodule or mass with homogeneous density. In certain cases, the nodules have a central apparent cavity $(5,6)$. These pulmonary lesions are considered to be benign tumors and primarily occur in middle-aged woman who have no history of smoking. The size of these lesions usually varies, typically ranging between 0.2 and $10 \mathrm{~cm}(7,8)$.

In the present study, the case of a 47-year-old female with an alveolar adenoma coexisting with intracranial vascular malformations is reported and a literature review was conducted, providing novel insights into the pathophysiology of this disease.

\section{Case report}

A 47-year-old female with no history of smoking underwent a routine medical examination prior to a hysteromyomectomy at the First Affiliated Hospital of Zhejiang University (Hangzhou, China) in November, 2010. A chest X-ray revealed a dense pulmonary nodule on the right side of the chest. Further examination using a computed tomography (CT) scan detected a 4-cm, low-density mass with air inclusions on the right lower lobe of the lung (Fig. 1A and B). A contrast-enhanced scan was produced with the attenuation of the postcontrast CT scan set to 20 Hounsfield units greater than the precontrast CT scan. The resulting image displayed a thin-rim of enhancement in the cyst wall, without internal enhancement (Fig. 1C). The initial diagnosis established by a radiologist was a pulmonary abscess; however, the patient did not present with any symptoms of infection, such as night sweats, weight loss, coughing or dyspnea. No abnormalities were detected in clinical and serological examinations, while pulmonary function was also normal.

In December 2010, a thoracoscopic segmentectomy was performed due to the possibility of low-grade malignancy of the pulmonary lesion. Gross examination of the resected lung tissues revealed a globular, pleural-based tumor, $4 \times 4 \mathrm{~cm}$ 
A

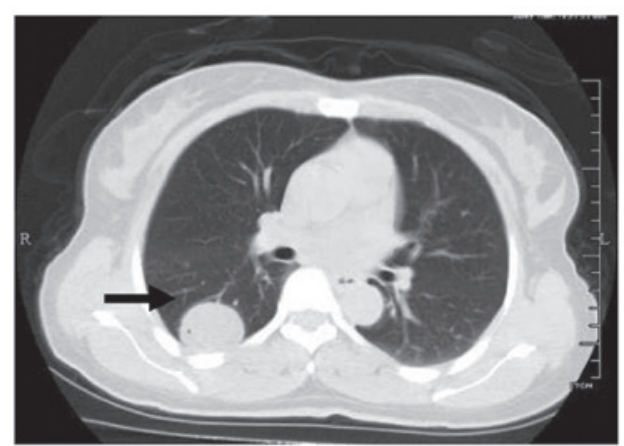

B

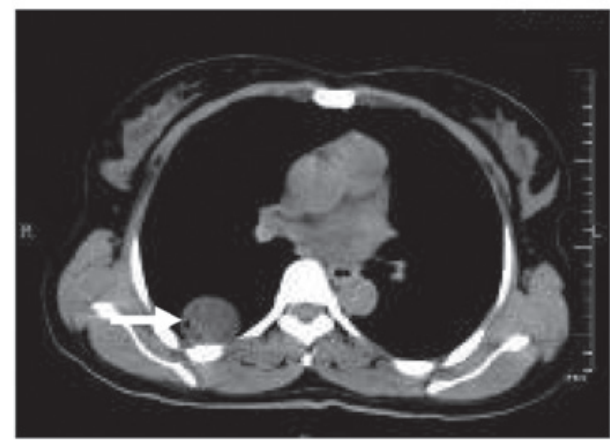

C

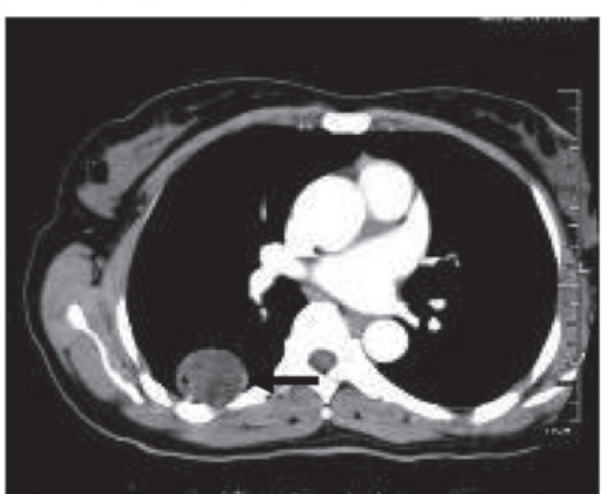

Figure 1. CT scans of the chest. A 4-cm, low-density mass with air inclusions on the right lower lobe was observed (arrows) (A) Lung window and (B) mediastinal window views. (C) Contrast-enhanced CT scan displayed a thin-rim of enhancement in the cyst wall but no internal enhancement. CT, computed tomography.

A

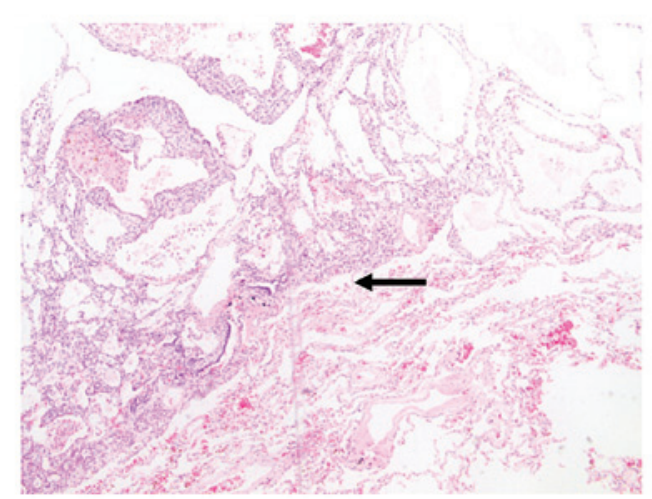

B

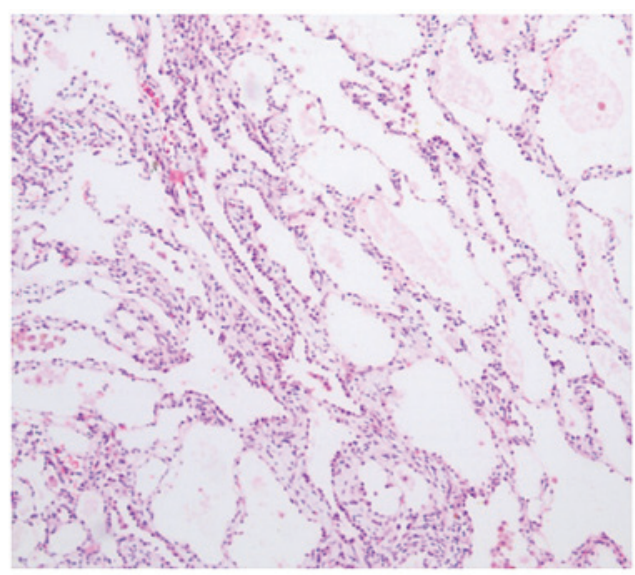

Figure 2. Microscopic analysis of the alveolar adenoma. (A) The tumor was a well-demarcated, multicystic mass (arrow) adjacent to lung parenchyma cells (hematoxylin and eosin stain; original magnification, x50). (B) The cystic spaces were filled with eosinophilic granular material and histiocytes, while the background stroma varied (hematoxylin and eosin stain; original magnification, $\mathrm{x} 100$ ).

in size, which was soft and pale grey. Microscopic analysis of the frozen resected tissue indicated that the tumor was a well-demarcated, non-invasive, multicystic mass (Fig. 2A) with spaces filled with eosinophilic granular material and histiocytes (Fig. 2B). The cystic spaces were lined by different types of epithelial cells, including flat, cuboidal and 'hobnail' cells. The alveolar lumina contained a small number of histiocytes with minimal cellular atypia, no mitosis and no invasive growth. Immunohistochemical analysis revealed the flat and cuboidal epithelia were positive for pan-cytokeratin (CK), CK7, CD10 and thyroid transcription factor 1 (TTF1; Fig. 3A-C), but negative for vimentin, calretinin and
CD31. In addition, the mesenchyme was reactive for smooth muscle antigen (Fig. 3D). The stroma contained spindle cells mixed with inflammatory cells, histiocytes, lymphocytes, erythrocytes and plasma cells. No Clara cells, mucous cells and APUD cells were identified. These findings indicated that the tumor was an alveolar adenoma.

A routine CT scan of the head demonstrated the presence of a lesion next to the right lateral ventricle. However, the patient displayed no adverse symptoms, including headache, nausea, blurred vision or impairment of limb movement. In addition, the patient had no previous history of seizures or hypertension. A magnetic resonance imaging scan of the 
A

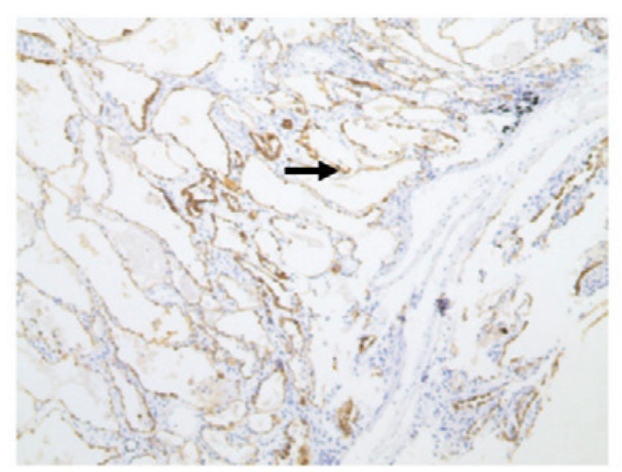

C

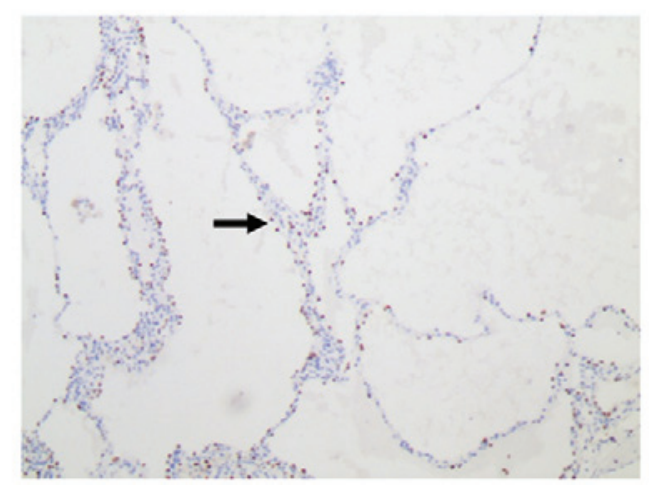

B

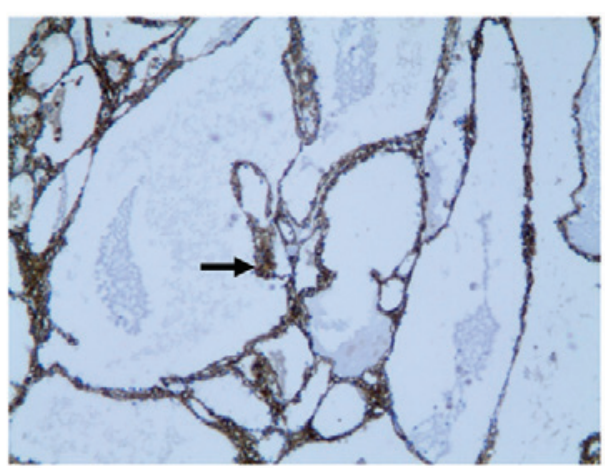

D

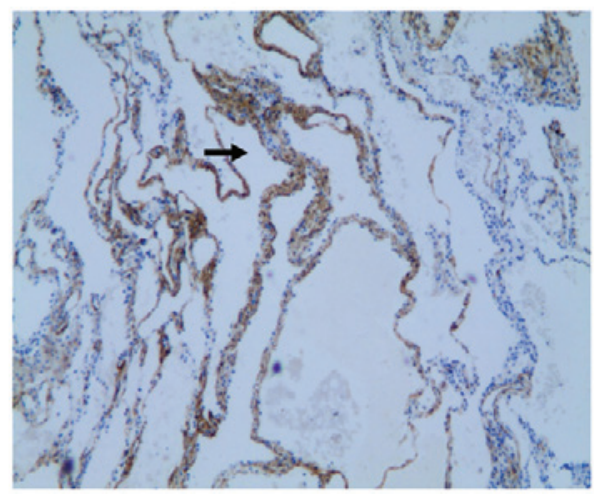

Figure 3. Immunohistochemical analysis of the alveolar adenoma. Staining revealed that the epithelial lining cells were positive for (A) cytokeratin 7 (hematoxylin and eosin stain; magnification, x50), (B) CD10 and (C) thyroid transcription factor 1, while (D) the mesenchyme was positive for smooth muscle antigen (hematoxylin and eosin stain; original magnification, x200). Arrows demonstrate cells with positive immunostaining.

A

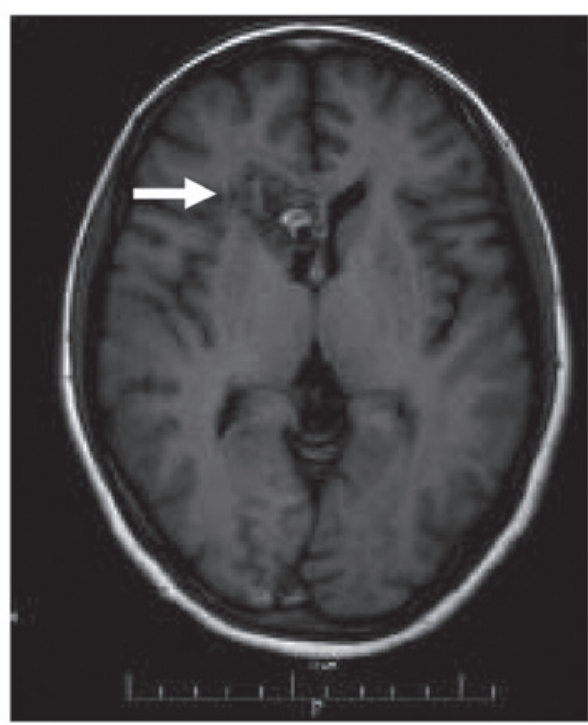

B

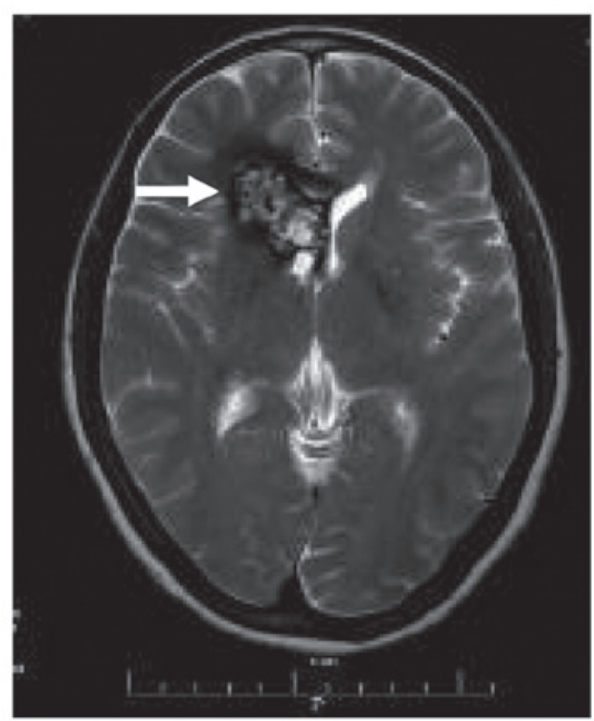

Figure 4. MRI scans of the head. (A) MRI scan revealing a 3.5x1.7- $\mathrm{cm}$ mixed signal lesion adjacent to the anterior horn of the right lateral ventricle (arrow). The T1-weighted image demonstrated a heterogeneous pattern in the small punctate lesion with a combination of high and low signal intensity areas. No edema or mass effect were noted. (B) T2-weighted image displaying honeycomb-like structures (arrow). MRI, magnetic resonance imaging.

head revealed a $3.5 x 1.7-\mathrm{cm}$ lesion with mixed signals next to the anterior horn of the right lateral ventricle. A T1-weighted image demonstrated a heterogeneous, punctate pattern with a combination of high and low signal intensity areas; however, no edema or mass effect was noted (Fig. 4A). A T2-weighted image displayed the presence of honeycomb-like structures (Fig. 4B). The intracranial lesion was diagnosed as an arteriovenous malformation with hemorrhage.
Following consultation with a neurologist in the Department of Neurological Surgery (First Affiliated Hospital, Zhejiang University), a digital subtraction angiography (DSA) was performed. No apparent cerebrovascular malformation was observed on the DSA. Considering the benign nature of the intracranial lesion, as detected by DSA, and the potential risks associated with surgery and radiotherapy, the patient selected to undergo follow-up with observation only. No 
exacerbation of the intracranial lesion was noted during the 4-year follow-up period. Similarly, the postoperative course of the alveolar adenoma was uneventful, and there was no sign of recurrence 52 months after surgery. The study was approved by the ethics committee of the First Affiliated Hospital of Zhejiang University, and written informed consent was obtained from the patient prior to publication of the study.

\section{Discussion}

Alveolar adenoma of the lung is an extremely rare primary benign epithelial lesion involving the lung parenchyma, which can have an alveolar or papillary cellular architecture $(1,3,4)$. Determining of the exact number of alveolar adenoma cases is difficult, since these tumors are often confused with other rare benign lung tumors. The first alveolar adenoma case was reported by Yousem and Hochholzer in 1986 (2). To the best of our knowledge, the present study is the first report of the coexistence of alveolar adenoma with intracranial arteriovenous malformations.

Alveolar adenoma seldom causes symptoms. In a limited number of cases, patients have presented with chest pain or chronic cough (9-11). Only one previous study reported the admission of an alveolar adenoma patient to the hospital with severe dyspnea (12). Most alveolar adenoma patients are 40-60 years old, with a slight female predominance $(1,2,8)$. The youngest patient ever reported, to the best of our knowledge, was a 24-year-old male, who presented an 18x17-mm nodule on the lower lobe of the left lung (13). Although patients usually present with a single lesion, a previous study described a case with three nodules on the two sides of the lung (1).

Alveolar adenomas typically grow slowly; however, a number of studies reported a sudden increase in size similar to malignant tumor growth $(5,8)$. Although alveolar adenomas have no distinct imaging features, the contrast-enhanced CT scan performed in the present study displayed enhancement around a thin-rim of the nodule. Similar features have been identified in tuberculoma patients (14), as well as a previously-reported alveolar adenoma case (1).

From a diagnostic standpoint, a 'lung mass' is defined as an abnormal spot in the lungs with a size of $>3 \mathrm{~cm}$. The mass is considered to be a bronchogenic carcinoma until proven otherwise (15). In the present case, the pulmonary lesion identified on the CT scan was $4 \mathrm{~cm}$ in diameter and, therefore, larger than the prognostic threshold of $3 \mathrm{~cm}$. For this reason, the lesion was removed by surgical lobectomy for diagnostic and therapeutic purposes.

Structurally, alveolar adenoma is comprised of epithelial and mesenchymal tissues, which can be identified by histological analysis (3-5). Upon examination using light microscopy, alveolar adenoma appears to be sharply demarcated from the surrounding tissue, but with lack of encapsulation, similar to the microcystic-like spaces that predominate the lesion. The cystic spaces contain a clear cellular fluid, as well as histiocytes, erythrocytes and foamy macrophages (4). In the current case, these cystic spaces were lined with a single layer of epithelial cells, which were mostly cuboidal or 'hobnail' in appearance.

Previous immunohistochemical studies have demonstrated that alveolar adenoma epithelia are positive for markers including pan-CK, epithelial membrane antigen and TTF-1 (16). Additionally, epithelia are positive for type II pneumocyte markers, indicating that the epithelial layer is derived from type II pneumocytes (16). In cases where the epithelial layer is flattened in shape and resembles the endothelium, the absence of immunostaining for CD31 or CD34 vascular markers may help to distinguish alveolar adenoma from other pulmonary lesions $(17,18)$.

The standard diagnosis of thoracic diseases is challenged by varied pathological findings in patients. Using clinical and histological findings, the current World Health Organization Classification of Tumors (19) states that benign adenomas of the lung include mucous gland, mucinous cyst, pleomorphic, alveolar and papillary adenomas. Known histological markers must be used for the differential diagnosis of benign alveolar tumors from malignant tumors, including pulmonary adenoma, sclerosing hemangioma, lymphangioma, atypical adenomatous hyperplasia and bronchoalveolar carcinoma. For instance, positive immunostaining for CK in the peripheral epithelial cells and negative immunostaining for vascular markers can distinguish alveolar adenomas from lymphangiomas (4).

However, diagnostic discrimination between alveolar adenoma and bronchioloalveolar adenocarcinoma has been proven to be difficult, since the two tumors exhibit positive immunoreactivity for TTF-1, surfactant and epithelial markers $(4,11)$. Therefore, for an unambiguous diagnosis of alveolar adenoma, the findings of immunohistochemical staining and gross structural analysis are also required. The well-circumscribed growth pattern, lack of lepidic growth and cellular atypia of alveolar adenoma distinguishes it from the other carcinomas. Considering that alveolar adenoma has a characteristic multicystic histology and often resembles the normal lung parenchyma, pathological analysis of the entire tumor resection is required for a clear-cut diagnosis $(5,10,20)$.

Intracranial arteriovenous malformations are congenital vascular lesions usually identified in patients with an age of 20-40 years (21). The typical clinical presentation includes hemorrhage, seizures, progressive neurological deficit or headaches (21). In the present study, the patient experienced no neurological symptoms and no apparent cerebrovascular malformations were detected by DSA. A neurologist diagnosed the intracranial lesion as an occult arteriovenous malformation based on previously-reported features (22). Although surgery is the recommended treatment modality (23), the patient selected to undergo long-term follow-up with clinical observation only, considering the risks associated with surgery and radiotherapy. During the 3-year follow-up period, no exacerbation of the intracranial lesion was observed.

In conclusion, alveolar adenoma is an extremely rare disease, which requires careful diagnosis and differentiation from a solitary pulmonary lesion. Surgical resection remains the best solution for the majority of patients. To the best of our knowledge, no recurrence of alveolar adenoma has ever been reported $(1-6,8-11,20,24)$. The present study is the first to report a case of incidental identification of alveolar adenoma with intracranial vascular malformations. The accurate diagnosis and appropriate treatment provided the patient with an improved prognosis following surgical lobectomy. Finally, 
the present study provides new insight into the diagnosis of alveolar adenoma.

\section{Acknowledgements}

This study was supported by a grant from the Zhejiang Provincial Education Department, China (no. Y200804939).

\section{References}

1. Fujimoto K, Muller NL, Sadohara J, Harada H, Hayashi A and Hayabuchi N: Alveolar adenoma of the lung: computed tomography and magnetic resonance imaging findings. J Thorac Imaging 17: 163-166, 2002.

2. Yousem SA and Hochholzer L: Alveolar adenoma. Hum Pathol 17: 1066-1071, 1986.

3. Sak SD, Koseoglu RD, Demirag F, Akbulut H and Gungor A: Alveolar adenoma of the lung. Immunohistochemical and flow cytometric characteristics of two new cases and a review of the literature. Apmis 115: 1443-1449, 2007.

4. Burke LM, Rush WI, Khoor A, et al: Alveolar adenoma: a histochemical, immunohistochemical and ultrastructural analysis of 17 cases. Hum Pathol 30: 158-167, 1999.

5. Kondo N, Torii I, Hashimoto M, et al: Alveolar adenoma of the lung: a case report. Ann Thorac Cardiovasc Surg 17: 71-73, 2011.

6. Saito EH, de Aaraujo LR, Carneiro LH, de Oliveira Neto AA, Correa JC and Teixeira LS: Alveolar adenoma. J Bras Pneumol 32: 267-269, 2006.

7. Bhavsar T, Uppal G, Travaline JM, Gaughan C, Huang Y and Khurana JS: An unusual case of a microscopic alveolar adenoma coexisting with lung carcinoma: a case report and review of the literature. J Med Case Rep 5: 187, 2011.

8. Wang X, Li WQ, Yan HZ, et al: Alveolar adenoma combined with multifocal cysts: case report and literature review. J Int Med Res 41: 895-906, 2013.

9. Cakan A, Samancilar O, Nart D and Cagirici U: Alveolar adenoma: an unusual lung tumor. Interact Cardiovasc Thorac Surg 2: 345-347, 2003.

10. Nosotti M, Mendogni P, Rosso L, et al: Alveolar adenoma of the lung: unusual diagnosis of a lesion positive on PET scan. A case report. J Cardiothorac Surg 7: 1, 2012.
11. Hartman MS, Epstein DM, Geyer SJ and Keenan RJ: Alveolar adenoma. Ann Thorac Surg 78: 1842-1843, 2004.

12. Petrella F, Rizzo S, Pelosi G, Borri A, Galetta D, et al: Giant alveolar adenoma causing severe dyspnoea. J Thorac Oncol 5: 1088-1090, 2010.

13. De Rosa N, Maiorino A, De Rosa I, Curcio C, Sellitto C and Amore D: CD34 expression in the stromal cells of alveolar adenoma. Case Rep Med 2012: 913517, 2012.

14. Sakai F, Sone S, Maruyama A, et al: Thin-rim enhancement in Gd-DTPA-enhanced magnetic resonance images of tuberculoma: a new finding of potential differential diagnostic importance. J Thorac Imaging 7: 64-69, 1992.

15. Gould MK, Fletcher J, Iannettoni MD, et al: Evaluation of patients with pulmonary nodules: when is it lung cancer?: ACCP evidence-based clinical practice guidelines (2nd edition). Chest 132 (Suppl): 108-130, 2007.

16. Halldorsson A, Dissanaike S and Kaye KS: Alveolar adenoma of the lung: a clinicopathological description of a case of this very unusual tumour. J Clin Pathol 58: 1211-1214, 2005.

17. Cagle PT, Allen TC and Fraire AE: Alveolar adenoma. Atlas of Neoplastic Pulmonary Disease: 19-21, 2010.

18. Limmer S, Krokowski M and Kujath P: Pulmonary lymphangioma. Ann Thorac Surg 85: 336-339, 2008.

19. Travis WD, Brambilla E, Müller-Hermlink HK and Harris CC (eds). Pathology and genetics. Tumours of the lung, pleura, thymus and heart. In: World Health Organization Classification of Tumours. IARC Press, Lyon, 2004.

20. Hamid Kazerouni A and Chetty R: Alveolar adenoma of the lung. Diagnostic Histopathology 19: 311-313, 2013.

21. Fleetwood IG and Steinberg GK: Arteriovenous malformations. Lancet 359: 863-873, 2002.

22. Lobato RD, Perez C, Rivas JJ and Cordobes F: Clinical, radiological and pathological spectrum of angiographically occult intracranial vascular malformations. Analysis of 21 cases and review of the literature. J Neurosurg 68: 518-531, 1988.

23. Mossa-Basha M, Chen J and Gandhi D: Imaging of cerebral arteriovenous malformations and dural arteriovenous fistulas. Neurosurg Clin N Am 23: 27-42, 2012.

24. Nakamura H, Adachi Y, Arai T, et al: A small alveolar adenoma resected by thoracoscopic surgery. Ann Thorac Surg 87: 956-957, 2009. 